\title{
Do not resuscitate decisions: discussions with patients
}

\author{
Stanley G Schade and Hyman Muslin University of Illinois at Chicago
}

\section{Authors' abstract}

The problem of psychological pain caused by discussions of do not resuscitate status with patients is addressed. Case histories of patients with such distress are given. We propose that not all patients should be informed of their do not resuscitate status, that the information about such status be given incrementally, and that the giving of further information be guided by the patient's reaction to earlier information. While some affirm the duty of the physician always to inform the patient about his or her do not resuscitate status, we affirm the duty of the physician to determine whether the patient wishes to enter into this discussion.

\section{Introduction}

The development of resuscitation methods such as external cardiac massage and easily applied ventilatory techniques has led to the need to specify under what circumstances these procedures should be applied. Medical personnel recognise that it is not appropriate to apply these methods routinely to patients who have suffered cardiovascular collapse but whose illness is not compatible with further survival. Although it is recognised that patients have a right to refuse these procedures, the psychological issues involved in discussion of these matters with patients in these circumstances have not been adequately addressed. This paper is an attempt to highlight the issues and the dilemmas in physician-patient interactions during the terminal stages of the patient's life.

Present day discussion of this subject is rooted in the concept of the patient as an autonomous decisionmaker and the notion that the dignity of the patient as an individual requires full participation in the information relating to diagnosis and treatment. The guiding principle in dealing with patients is the recognition that actual authority over the patient never belongs with the physician (1). From this view, physicians serve as consultants to patients advising them as to their options and helping them to make their decisions.

\section{Key words}

Do not resuscitate orders; paternalism; patient autonomy versus psychological pain.
This policy is justified in many medical decisionmaking processes. In concert with this approach, medical practitioners and their advisors have concluded that a patient facing imminent death should be informed so that he or she can also participate in the decision as to whether to have respiratory and cardiac support when these functions cease $(1,2)$.

Although there is a logical continuity which seems to require this final consenting process, we have concluded that there is a marked emotional discontinuity which requires re-thinking of this process (3). During the past seven years we have observed patients whose emotional well-being has been harmed by this final consenting process.

In psychiatric practice, the physician often delays or avoids delivering to his patients sensitive information in the process of treatment, awaiting the proper moment for delivery of the information. The timing of delivery of this information depends upon the physician's empathic understanding of the state of preparedness of the patient and the importance to the patient of acquiring the information. In dealing with a dying patient the physician often has little or no opportunity to offer information in any useful or meaningful way to the patient. The physician is faced with a patient whose illness is of such a nature that discussions of resuscitation procedures simply add to the agony of the dying process. In this situation, should the physician be obliged to advise his patient and to ask his patient to consent to the withholding of resuscitation procedures?

It has been argued that it is not the patient who suffers from this process but the physician. This argument contends that the physician is reluctant to give up his role as benefactor to the patient, reluctant to admit his defeat to the patient. The contrary argument has been advanced that physicians avoid these discussions because of their concern for their patients' psychological well-being $(3,4)$. The following cases illustrate some of the psychological problems which may be associated with a do not resuscitate discussion.

\section{Patient histories}

Patient 1:

The patient was a 49-year-old former telephone 
employee with multiple myeloma which had failed to respond to treatment with melphalan and prednisone, and hypercalcaemia was a recurring problem. In an attempt to control the disease high doses of alternateday prednisone therapy were initiated. At this time one of the authors approached the patient for the purpose of an exploratory discussion of a do not resuscitate decision. The physician introduced the subject by remarking that at some point in the treatment of multiple myeloma the disease becomes more aggressive and difficult to treat. While this situation had not occurred in this particular patient, the physician did want to explore with the patient the decision she would want to make in the event that the disease did become uncontrollable. The physician said that one question that came up was related to chest compression or artificial respiration should such a patient stop breathing or should the heart stop. The patient seemed to tolerate the discussion since it was rendered in this indirect manner, the physician suggesting that this was a common type of discussion to have with patients with the type of disease that she had, and that such decisions were not contemplated at the present time, although they might take place at some indefinite future date.

The patient said that she would not wish to have compression of her chest to restart her heart, particularly if the physician thought that such a procedure would be futile. She also thought that she would not want to be informed that such a decision had been made about her treatment.

While the patient did not give much indication of discomfort with this discussion, she did say that she was using 'imaging' therapy to help herself to treat her myeloma. She would do this by imagining that the tumour was dissolving away.

Over the next several days, following this discussion, other physicians noted that the patient appeared to have undergone a transformation. She now revealed frequent episodes of agitation and complained that her imaging therapy was no longer effective. The agitation was such that the doctor on call felt that she needed sedation. Her conversation now revealed a derailing of her thought processes; she trembled and breathed rapidly. Another physician commented to the doctor who had held the discussion with her that the patient's conversation thereafter was flooded with death imagery.

The patient presents an example of a discussion in advance of the time in which a decision would have to be made, which caused psychological discomfort and disarray.

\section{Patient 2:}

The patient was a 50-year-old male who had advanced chronic lymphocytic leukaemia which was no longer responsive to chemotherapy. He had severe anaemia and thrombocytopaenia requiring support with blood products. When a pleural effusion developed, it was necessary to place a tube for drainage. At this time, the attending physician felt that 'the patient's disease was so advanced that resuscitative procedures would be futile in the event of cardiac arrest. The resident physician discussed with the patient and the family the do not resuscitate decision and obtained the consent from the patient and the family for this order. On the following day when the attending physician talked with the patient, the patient appeared to have no recollection of the details of the discussion. He only recalled that he believed the physicians felt he was being un-co-operative in some way. He said he had been observing himself closely since then to make sure he was being co-operative with his physicians. It seemed that the do not resuscitate discussion had in some way been very disturbing to the patient, but that he had masked the import of the discussion with a memory that told him that his physicians had been upset by his un-co-operative behaviour. The memory of a painful event had been transformed into a less painful memory, although it was still clear that psychological anguish was produced. The patient had experienced the discussion as a rebuke.

\section{Patient 3:}

The physician discussed the problem of do not resuscitate decisions with a patient with acute leukaemia who was going into remission. The physician and patient had had a long conversatio about the difficulties experienced by the patient after learning of the diagnosis of leukaemia and the uncertainty that surrounded the patient's future course. At the end of the conversation the physician introduced the subject of resuscitation by saying that patients with leukaemia had relapses and that after multiple attempts at remission the patient might experience cessation of heart or pulmonary action at which point attempts to resuscitate were generally futile. The physician wanted to know from the patient whether he would want to give the physician the permission ahead of time not to attempt resuscitation. The patient wanted to think that over and declined the offer to give that authority into the physician's hands. Later, during an interview with the consulting psychiatrist the patient told the psychiatrist that indeed he had given to his doctor the permission to write a do not resuscitate order if the physician felt that this order were appropriate.

This illustrates that patients may be willing to acknowledge acceptance of a do not resuscitate decision to care-givers other than the physicians whom they perceive as directly responsible for writing the order.

\section{Patient 4:}

The patient was a 48-year-old male who had had multiple myeloma for ten years. Throughout this period of time the disease had responded to various types of therapy. With each progression of the disease, the patient had experienced severe anxiety. It was 
difficult for him to relate to those caring for him and he often complained about them. His physician had, throughout the course of his illness, carefully explained to him the various forms of treatment which were being employed.

Eventually, the patient developed recurrent episodes of hypercalcaemia which were unresponsive to chemotherapy for the myeloma but which were briefly controlled by intravenous plicamycin. The physician was certain that the patient's death was imminent and did not want to resuscitate since the patient had extensive bone disease and any attempt to do so would have been futile. However, because of the patient's known reaction to past information concerning progress of the disease the physician did not want to advise the patient about his do not resuscitate status. He did discuss it with the patient's brother and sister-in-law and they both agreed that no attempt at resuscitation should be made if the patient suffered cardiac arrest. They also agreed that the patient not be informed of the do not resuscitate order. However, they did wish the hypercalcaemia to be treated as long as possible and any infections to be treated aggressively. During the patient's last hospitalisations he was relatively calm and received visits from his son and mother who travelled long distances to see him. The physician did not reveal to the patient his do not resuscitate status. During this time, the physician did feel uneasy because he had not advised the patient of the imminence of his death. However, the patient showed no evidence of distress during this time and the immediate family were well satisfied with his care and with this particular approach.

\section{Patient 5:}

The patient was a 71-year-old woman who was admitted to hospital for the treatment of a relapse of acute myeloblastic leukaemia. On admission the patient was briefly septic and was hypotensive. She responded initially to antibiotic therapy. Her physician asked her whether she wished to undergo chemotherapy in the hope of a remission and discussed the issue of resuscitation. The patient was willing to undergo chemotherapy and said that she would even be willing to have intubation to sustain her respiration for a period of time in case her conditon required this. Her physician told her that in his experience if patients with her disease state suffered cardiac arrest that it was futile to try to start the heart again by cardiac compression. He asked her if she objected to having an order written which would state that she did not desire to have cardiac resuscitation in case of cardiac arrest. The patient agreed to this type of order.

Three days later on a subsequent visit to the patient the physician found that she had no recall of the do not resuscitate decision. She did recall discussing with the physician some aspects of the care of her retarded daughter at home. Any memory, however, of the do not resuscitate decision seemed to have been lost.
Patient 6:

The patient was a 42-year-old female who had been undergoing treatment for multiple myeloma for the previous four years. She had initially presented with back pain at which time a diagnosis of multiple myeloma was made. She responded well to treatment with melphalan and prednisone but after three years experienced advancing disease with pain requiring treatment with oral morphine.

She experienced leukopaenia and developed severe pneumonia requiring admission to the medical intensive care unit. Her doctors there discussed with her the possibility of suffering cardiopulmonary arrest, and she consented to the placing of a do not resuscitate order. Following recovery from the pneumonia she was returned to the medical floor where one of her physicians discussed with her the significance of the do not resuscitate discussion. She said she was quite happy with the discussion and pleased that the doctors in the intensive care unit had informed her about the seriousness of her condition. She related that she was a religious person with a strong Roman Catholic faith. She believed that there was an afterlife in which she would participate. When the doctor asked whether she thought that doctors in general should discuss with their patients do not resuscitate orders, she replied that she felt that with some patients it would not be advisable, but with her it had been the correct thing to do.

Following this episode the patient began to voice to her care-givers strong fears of her approaching death. She had great difficulty communicating these fears to members of her immediate family who tended to deny the presence of her disease. At home she became confused, perhaps related to the dosages of morphine that she was taking, and required re-admission to the hospital. On re-admission she was quite depressed and frightened about her approaching death. When she talked to one of her doctors again she told him that she now thought it was inadvisable for her to have had the discussion about do not resuscitate orders. She stated that if things became clinically worse for her in the future that she would not want to be advised about her do not resuscitate status. She said that it would be wrong to remove hope from her, that this was the most important thing that she now had.

\section{Patient 7:}

The patient was a 63-year-old man who was in the hospital for one of his many admissions during his illness with myeloma when a staff physician approached him to discuss the progress of his illness and his wishes about resuscitation. In response to the inquiry about resuscitation, the staff physician reported, the patient did not appear to recognise the gravity of his situation and appeared confused. In his interview the psychiatric consultant observed that the patient not only was in pain and unable to find a comfortable place in bed but was speaking rapidly and tangentially about a variety of matters, none of which 
were related to his illness. When the psychiatrist inquired about his view of the illness, he appeared dazed and, with considerable anxiety, he referred him to his brother with whom he had lived for the past several years. When the psychiatrist persisted several times in his attempts to evoke responses from the patient about his illness, the patient changed the subject and finally insisted he was too tired to discuss anything. Over the rest of his hospital stay he managed to avoid any direct contact with the psychiatrist.

\section{Discussion}

One of the most important and delicate tasks of the physician is informing the patient about the nature of his or her illness. This task is, of course, especially critical when the illness is a serious one, particularly when it is known that the outcome is frequently fatal. The physician may approach this task by discussing the disease and its implication in general terms, observing the patient's reactions, and leading the discussion according to these reactions. By this process the physician judges how much information to give at any one time and how to adjust the flow of information so that the patient is not psychologically overwhelmed. A careful and cautious delivery of information, guided by patient feedback, improves the chances that the patient can absorb the information with the least emotional distress.

As an example, the physician would not approach a patient with the following: 'You have acute leukaemia. Your survival is estimated at 23 months. Your chances of remission with the first trial of chemotherapy are 80 per cent, etc'. Rather the physician might approach such a patient with the statement: 'You have a blood disease. This disease will require hospitalisation for treatment, etc,' with further information being delivered in reaction to the patient's responses.

This method of informing patients does not attempt to slight the patient's role or autonomy; rather, the attempt is to maintain self-determination by encouraging calm and rational participation in decision-making.

Paradoxically, in one of the potentially most traumatic instances of patient-doctor communication, that dealing with a patient with advanced malignant disease who is about to die, the physician may feel required by the mores of modern medicine to request directly from his patient consent for resuscitation procedures not to be instituted if heartbeat or respiration cease.

Many do not resuscitate protocols require discussion with the competent patient (5). When the procedure is of no medical benefit, Tomlinson and Brody have stated that "communication with the patient or family should aim at securing an understanding of the decision the physician has already made' (6). On the other hand, these authors have also stated that in the absence of medical benefit 'there would be no need for discussion, since the justification for the order would not rest on information about the patient's values or preferences' (6). Spencer (4) has also argued against discussion of the decision on the grounds that such information would be too distressing.

In short, modern medicine is ambiguous about the necessity for informing patients about do not resuscitate decisions, although the general understanding amongst physicians is that such discussions are necessary (7), and physicians often feel compelled to carry out these discussions, whereas in other contexts they would tailor their information so as to be as gentle as possible.

The above case studies show that a legitimate concern underlies the physician's desire to avoid this discussion; namely, the concern that the discussion will inflict useless and unnecessary suffering on the patient. Although not informing the patient about this decision may make the physician uneasy, it would seem a reasonable duty in some instances for the physician to take the responsibility for this order without informing the patient in order to preserve the patient's psychic equilibrium and to minimise the suffering associated with the patient's final separation from life and loved ones.

Our concern that patients' psychological comfort be a factor in discussions with patients is often seen as a remnant of a paternalistic, non-egalitarian tradition which arouses deep suspicions when presented abstractly. The opposing argument rests on the dominant tradition of the Enlightenment which requires that all information about a patient be shared with that pritent. In this view, the patient has an absolute right :o information about his or her imminent death which no consideration of comfort should override. Adherence to either position is more a matter of intuitive belief than of logical argument. In essence, one side affirms the duty of the physician to inform the patient about do not resuscitate orders, while we, on the other side, affirm that the duty of the physician is first to ascertain whether the patient wishes to enter into such a discussion.

Based on our concern with patient comfort we would propose that there are instances in which physicians might write a do not resuscitate order for a patient without so informing the patient. This would be a decision made by the physician after careful consideration of the patient's ability to cope with such information and after consideration of whether the particular doctor-patient relationship required such disclosure. All would depend on the nature of the data received by the physician from the patient. Some patients might welcome and benefit from knowing about a do not resuscitate decision; many, however, would be best served by not being explicitly told of the order. Clearly, when the physician undertakes such a responsibility, he should state his thinking and reasoning in the hospital chart, and he should also discuss his views with the patient's significant relatives, at least informing them of his decision and obtaining their agreement to such a process.

It is important to understand that the writing of such 
an order does not preclude the physician from undertaking support of the patient in every other regard. For instance, a patient with advanced multiple myeloma resistant to chemotherapy might receive extensive treatment for hypercalcaemia, infection, and pain in spite of the fact that the physician had entered a do not resuscitate order for the patient. Withdrawal of other forms of support such as treatment of hypercalcaemia might become appropriate and might be permitted as a merciful form of death and release from suffering. However, the non-treatment of hypercalcaemia in this instance is not required by the presence of a do not resuscitate order. All the capacities of the physician and the hospital staff may be exerted to preserve the patient's life short of the resuscitation procedures when the heart stops and the respiration stops.

It may be objected that the problems here discussed could all be obviated by an early discussion with the patient about his or her desires regarding resuscitation. Although it is true that an early discussion might be less emotionally distressing to the patient since the event can be seen as distant in time, it may nevertheless be questioned whether early discussion is appropriate. For instance, when a patient is first informed of the diagnosis of malignant disease, it is often of principal importance to the physician to encourage hope that life might go on for long periods of time even in the presence of malignant disease. It is difficult to introduce a do not resuscitate discussion in talking to a patient when the physician is trying to encourage hope and assurance of support. More importantly, the physician may feel that a patient with a colon carcinoma with a metastatic nodule in the liver may be ill-served by a do not resuscitate order when the patient is feeling well. In the unlikely event that a myocardial infarction should occur with a transient arrhythmia, a decision not to resuscitate may deny the patient months of satisfying life.

It thus may seem best medically to defer the decision on resuscitation to a point in time when the disease is clearly so advanced that resuscitation would be futile. However, at that time to discuss it with a patient because of a concern for patient autonomy takes on the appearance of an over-concern for the institutionalised forms of morality while ignoring painful, psychological realities. These discussions are painful for the patient, they leave in their wake psychological distress and anxiety, and they may prevent a peaceful exit for the patient who, while surely knowing that death is near, is yet permitted the hope that it is not yet.

Stanley $G$ Schade MD is Professor of Medicine in the Department of Medicine, University of Illinois at Chicago and Staff Physician at the West Side Veterans Administration Hospital, Chicago, Illinois. Hyman Muslin MD is Professor of Psychiatry in the Department of Psychiatry, University of Illinois at Chicago, Chicago, Illinois.

\section{References}

(1) Ruark J E, Raffin T A, Stanford University Medical Center Committee on Ethics: Initiating and withdrawing life support. New England journal of medicine 1988; 318:25-30.

(2) Angell M. Respecting the autonomy of competent patients. New England journal of medicine 1984§ 310:1115-1116.

(3) Muslin H, Schade S. On the do not resuscitate policy Perspectives in biological medicine 1988; 31:285-290.

(4) Spencer S S. 'Code' or 'no code': a non-legal opinion. New England journal of medicine 1979; 300:138-140.

(5) Bedell S E, Pelle D, Maher P L, Cleary P D. Do-notresuscitate orders for critically ill patients in the hospital. Fournal of the American Medical Association 1986; 256:233-237.

(6) Tomlinson T, Brody H. Ethics and communication in donot-resuscitate orders. New England journal of medicine 1988 ; 318:43-46.

(7) Farber, S J. [letter to the editor]. New England journal of medicine 1988; 318:1757.

\section{News and Notes}

\section{Symposium on torture}

An international symposium on Torture and the Medical Profession will be held at the University of Troms $\varnothing$, Norway from June 5-7 next year.

Topics will include: Ethical problems in relation to the medical profession and torture; Historical perspectives - given by doctors who themselves have been imprisoned in concentration camps; Medical, ethical and legal aspects - theoretical considerations; Testimonies by torture victims, who have witnessed medical doctors involved in the procedure, and Human rights - international aspects, including international co-operation against medical torturers.

For more information and/or to register contact: Jørgen Cohn, MD, Professor of Pediatrics, Department of Pediatrics, University of Troms $\varnothing$, Post Office Box 2415, N-9012 Troms $\varnothing$, Norway. Telephone: 083 44734, Telefax: 08380850. 